\title{
Die Historizität der Verdatung: Konzepte, Werkzeuge und Praktiken im 19. Jahrhundert
}

\author{
Christine von Oertzen
}

The Historicity of Data: Concepts, Tools, and Practices During the Nineteenth Century

This essay explores the use of the concept of "data" during the nineteenth century. It traces the development of manual data driven research decades before the introduction of Hollerith machines and electronic computers. Census statisticians in late-nineteenth century Prussia employed moveable paper tools to assemble numerical information in novel ways; their actions fundamentally recast processes of compilation. The paper considers the epistemic impact of moving data inscribed on tons of paper, reconstructing the logistics of a circulatory compilation system spread across the Prussian capital. The movement of data in Wilhelmine Berlin reached outward from the statistical bureau to encompass the homes of many women, enlisted in a minutelyorchestrated putting out system. The essay scrutinizes the social and intellectual requirements necessary to classify, sort, and aggregate the information on the loose paper slips fast and without error. For all of their inventiveness, Prussian statisticians were neither the first nor the only ones to gather, process and visualize data in imaginative ways. As this essay demonstrates, many so-called "Baconian" sciences developed similar tools and practices to those of Prussian statisticians. Making and moving data sparked historical sensitivity in disciplines well beyond population statistics, from taxonomy to paleontology, yielding unexpected revelations.

Keywords: Data history, Population statistics, Prussian census, Manual practices, Material culture

Der Beitrag nimmt den heute allgegenwärtigen Begriff „Daten“ als historische Kategorie in den Blick. Er geht der langsamen Verbreitung des Wortes unter Statistikern im 19. Jahrhundert nach und untersucht die materielle Kultur derjenigen Konzepte und Praktiken, die mit seiner Verwendung einhergingen. Am Beispiel der preußischen Volkszählung legt der Beitrag mit diesem Vorgehen bislang unbeachtete Genealogien datengetriebener Forschung frei: Nicht erst Computerspezialisten des 20. und 21. Jahrhunderts, sondern Wissenschaftler des 19. Jahrhunderts machten sich den Begriff für die Produktion streng abstrahierter, numerischer Information zunutze. Sie entwickelten hochflexible Aufbereitungsverfahren von Massenerhebungen, lange bevor Maschinen wie das Hollerith-System oder der Computer die Kompilierung per Hand ersetzten. Bei der preußischen Volkszählung war das Werkzeug dieser „Verdatung” die sogenannte Zählkarte, ein beweglicher Papierträger, der ein komplexes System der Auswertung erzwang. Der Beitrag rekonstruiert die Logistik dieses Verfahrens und verfolgt die durch ganz Berlin zirkulierenden Papiermassen. Er analysiert die Organisation der meist weiblichen, in Heimarbeit beschäftigten Auszähler und ermisst die sozialen und intellektuellen Anforderungen, die erforderlich wurden, um die auf den beweglichen Zählkarten eingeschriebenen Informationen möglichst schnell und fehlerlos in numerische Aggregate umzuwandeln. Der Beitrag argumentiert, dass die Verdatung nicht nur in der Bevölkerungsstatistik zu neuen Darstellungsformen und Erkenntnissen führte, sondern auch die Forschungspraktiken etlicher anderer "baconischer" Wissenschaften prägte. Von der Nationalökonomie über die Taxonomie bis zur Paläontologie stärkte das Sehen und Verarbeiten von „Daten" historische Sensibilitäten und führte zu überraschenden, epochalen Einsichten. 
Das Phänomen „Big Data“ hat in den letzten Jahren zu verstärktem Nachdenken über datengesteuerte Methoden in der Forschung geführt, die lange vor der Digitalisierung existierten. Angeregt wird das historische Interesse an Datenpraktiken von Behauptungen, dass sich heute angesichts der riesigen Menge vorhandener Daten wissenschaftliche Hypothesenbildung gleichsam von selbst erledige (Anderson 2008). Den vorgeblich rein induktiven Charakter von Big Data in den Wissenschaften haben Science and Technology Studies wie auch die Wissenschaftsphilosophie inzwischen kritisch hinterfragt; durch deren Kritik ist die Rolle der Theorie in der Datenproduktion und -verarbeitung in den Blick gekommen (Leonelli 2008, 2016; Bowker 2000). Die Reflexion über „Big Data“ hat so als Analyse heutiger Forschungsverfahren Kontur gewonnen und „Daten“ selbst wie auch den Umgang mit ihnen zum Untersuchungsgegenstand gemacht. Eine historische Kontextualisierung des Begriffs und seiner Praktiken steht hingegen noch in den Anfängen.

Dabei sind „Daten“ in der Wissenschaftsgeschichte keine neue Kategorie. Bei der auf Kuhn folgenden Neuorientierung des Fachs von Theorien hin zu Praktiken haben sie eine zentrale Rolle gespielt (Aronova et al. 2017: 4-7). So verdeutlichten Bruno Latour wie später auch Hans-Jörg Rheinberger in ihren Studien zur experimentellen Laborpraxis, dass und wie Daten konstruiert und erzeugt werden: Latour führte vor, wie durch wiederholte und rasche Kondensierung einzelner „Inskriptionen“ empirische Befunde zu einfacheren, kompakten und visuell fassbaren „immutable mobiles“ werden (Latour 1987); Rheinberger verfolgte die Verdichtung von „Spuren“, „Zeichen“ und „Indizien“ zu beständigeren und aussagekräftigen „Sachverhalten“ oder möglichen Tatsachen, die dann zu anderen Daten in Beziehung gesetzt werden mit dem Ziel, Strukturen zu erkennen und diese zu visualisieren (Rheinberger 2011). In der Geschichte geisteswissenschaftlicher Wissenspraktiken, der wissenschaftlichen Beobachtung oder der Quantifizierung standen Daten mittelbar im Fokus. Dabei ging es um Fragen, wie der Überfluss an Informationen seit der frühen Neuzeit jeweils bewältigt wurde - sei es in Form von überbordenden Mengen an Büchern und Schriften, unerschöpflichen naturhistorischen Beobachtungen oder der bürokratischen Lawine von Tabellen und Zahlen. ${ }^{1}$

An diese Arbeiten anknüpfend rücke ich den Begriff „Daten“ im Folgenden direkt und explizit als semantische und historische Kategorie ins Rampenlicht. Ich werde nachzeichnen, mit welcher Bedeutung und welchem Nutzen der Begriff seit dem späten 18. und vor allem im 19. Jahrhundert jeweils eingesetzt wurde. Gleichzeitig frage ich, von welchen Methoden, Technologien und Praktiken seine Verwendung begleitet war. Bei dieser Historisierung des Begriffs und der Analyse seiner jeweiligen materiellen Kultur lassen sich bislang unbeachtete Genealogien datengetriebener 
Forschung freilegen. Nicht Computerspezialisten des 20. und 21. Jahrhunderts, sondern Vertreter verschiedenster Wissenschaften des 19. Jahrhunderts machten sich den Begriff "Daten“ und sein methodisches Potential in neuer Weise zunutze und läuteten damit das numerische Informationszeitalter ein, ein Zeitalter, dessen Geschichte von Ian Hacking, Theodore Porter, Alain Desrosières, Lorraine Daston und anderen als Epoche der Quantifizierung bzw. der probabilistischen Revolution beschrieben worden ist. ${ }^{2}$ Aus der Perspektive dieses Beitrags werden die Techniken der Kompilierung staatlicher Erhebungen als Datenpraktiken erkennbar, die sich mit dem Aufblühen des statistischen Denkens herausbildeten und hinter der „Lawine von gedruckten Zahlen“ (Hacking 1990) des 19. Jahrhunderts standen. Die theoretischen, konzeptionellen und medialen Grundlagen datengesteuerter Forschung, so lautet die These dieses Beitrags, wurden mithilfe neu entwickelter, manueller Verfahren der Aufbereitung gelegt, lange bevor Maschinen - wie das Hollerithsystem oder der Computer - die Kompilierung per Hand ersetzten, beschleunigten und veränderten.

Exemplarisch lässt sich dies an der preußischen Bevölkerungsstatistik zeigen, die um 1860 eine tiefgreifende Erneuerung erfuhr. Der preußische Zensus und seine Reform sind von Wissenschaftshistorikern wie auch Historikern untersucht worden und haben dabei jenseits der kritischen Aufarbeitung im Rahmen der Quantifizierungsgeschichte vor allem im Kontext universitärer Disziplinbildung (Nikolow 1994) und als Institutionengeschichte Kontur gewonnen, die sich an systemtheoretischen Ansätzen orientiert, um die behördliche Eigenlogik statistischer Wissensproduktion aufzuzeigen (Schneider 2013). Aufbauend auf und in Auseinandersetzung mit dieser Literatur geht es im vorliegenden Beitrag um eine an der Geschichte von Daten interessierte Analyse der materiellen Kultur des preußischen Zensus. Mein Anliegen ist es freizulegen, wie die preußische Volkszählung zu einem explizit von Daten getriebenen Unterfangen wurde. Ich rekonstruiere dabei zum einen, wann und wie die preußischen Statistiker den Begriff „Daten“ verwandten; zum anderen geht es mir darum, die Methoden und Techniken der Aufbereitung der umfangreichsten deutschen Massenerhebungen im 19. Jahrhundert als mediale Praktiken sichtbar zu machen. Daten haben auch in dem Sinne Masse, als dass sie notwendigerweise ein physisches Korrelat aufweisen (Edwards 2010). In diesem Fallbeispiel sind es Papierträger, auf denen sie eingeschrieben und gespeichert wurden. Diese Speicher haben insofern epistemische Funktion, als sie die konzeptionellen wie auch materiellen Rahmenbedingungen von Bearbeitung und Resultat wesentlich prägen. Einen wichtigen Referenzpunkt dieses Beitrags stellen daher Forschungen dar, die sich mit praxeologischen Forschungsansätzen, Papiertechnologien wie auch mit Aufschreibe- 
systemen seit der frühen Neuzeit befassen, in der Verwaltung wie in der Wissenschaft. ${ }^{3}$

Die grundlegenden Veränderungen, welche die Erzeugung der preußischen Bevölkerungsstatistik zwischen 1860 und 1871 erfuhren, lassen sich am besten als „Verdatung" bezeichnen. Ich verwende diesen Begriff hier nicht in dem Sinne, wie er heute zumeist eingesetzt wird, das heißt als Speicherung und Verarbeitung personenbezogener Daten. ${ }^{4}$ Mit dem Blick auf das 19. Jahrhundert dient mir der Begriff der Verdatung dazu, einen Prozess der konzeptionellen Neudeutung und praktischen Umwandlung bis dahin üblicher Erfassungs- und Aufbereitungstechniken zu beschreiben. In Erhebungsmaterial „Daten“ zu sehen und als solche zu bezeichnen, ging Hand in Hand mit der Entwicklung von neuartigen Methoden und Werkzeugen, mehr und präzisere Informationen zu sammeln, und das erhobene Material nicht nur aufzuaddieren, sondern in Bewegung zu bringen und in verschiedensten Kombinationen miteinander in Beziehung zu setzen, in Aggregate zu kompilieren und diese graphisch darzustellen. Zwischen 1860 und 1871, so die These dieses Beitrags, wurde aus dem statischen Erhebungs- und Auswertungsverfahren des größten deutschen Staates ein dynamisches, von Daten getriebenes Unterfangen, bei dessen Konzeption auch der Begriff "Daten“ selbst explizit Verwendung fand.

Um diesen Prozess der Verdatung zu verdeutlichen, gehe ich in fünf Schritten vor: In einem ersten skizziere ich, in welchem Sinne der Begriff "Daten“ in der Bevölkerungsstatistik des 19. Jahrhundert Verwendung fand. Im zweiten Schritt rekapituliere ich die wichtigsten konzeptionellen Grundzüge der Bahn brechenden preußischen Zensusreform der 1860er Jahre, die mit der Verwendung des Begriffs „Daten“ einhergingen. Drittens widme ich mich der technisch-medialen Umsetzung dieser Innovation und der Einführung der sogenannter „Zählblättchen“ bzw. „Zählkarten“, das heißt beweglicher Papierwerkzeuge, die neue Auszählungs- und Darstellungsformen der Bevölkerungsdaten ermöglichten und sich nicht nur in Preußen, sondern auch in den statistischen Ämtern anderer deutscher Länder und europäischer Staaten durchsetzten. Im vierten Schritt konzentriere ich mich auf die Zeit nach 1871 und lege dar, wie die Umsetzung der Reformen und die Ausbeutung des Erhebungsmaterials in Berlin konkret vor sich gingen. Besondere Aufmerksamkeit gebührt hier der Logistik des Umgangs mit den tonnenschweren Papiermassen, der Organisation der Arbeitskräfte, wie auch den mentalen Anforderungen, die erforderlich wurden, um die neuen beweglichen Formulare schnell und möglichst fehlerlos auszuwerten. Die preußische Art, Volkszählungsergebnisse zu generieren, erweist sich dabei als erstaunlich komplexe und zirkulierende, räumlich weit greifende Operation, die sich über ganz Berlin erstreckte. Denn um die Daten in Bewegung zu bringen und in kürzester Zeit sowie mit den 
geringsten Kosten akkurate Resultate zu erzielen, setzte das KöniglichPreußische Statistische Bureau auf ein Verfahren der Auswertung, das im Sinne Latours wesentlich von der Masse der Daten und ihren beweglichen Papierträgern „angetrieben“ wurde: Es vertraute seine Unterlagen und den überwiegenden Teil der Kompilationsarbeiten den Ehefrauen und weiblichen Verwandten seiner männlichen Hilfskräfte und Beamten in Heimarbeit an. Im fünften und letzten Teil weite ich meine Perspektive aus und erörtere, inwiefern die Verdatung empirischer Erhebungen im 19. Jahrhundert ein Phänomen war, das sich mitnichten auf die Bevölkerungsstatistik beschränkte, sondern sich auch in anderen Forschungspraktiken nachweisen lässt, angefangen mit der Botanik und Taxonomie über die Paläontologie bis zur Historischen Schule der Nationalökonomie. Ich schließe meinen Beitrag mit einigen kurzen Überlegungen, warum die Verdatung gerade für diese historisch orientierten Disziplinen im 19. Jahrhundert neue Impulse setzte und zu überraschenden Ergebnissen führte.

\section{Von Daten sprechen}

Der lateinische Begriff „datum“ (das Gegebene) fand im 17. Jahrhundert über die Mathematik und Theologie Eingang in die europäischen Landessprachen. Er wurde dort zunächst für die Bezeichnung von Prinzipien oder Tatsachen wie etwa biblische, Gott gegebene Wahrheiten verwandt, die jenseits der Empirie lagen. In der Mathematik verwies der Begriff auf eine Übereinkunft, die der Suche nach Wahrheit diente, aber selbst unverrückbar war (Rosenberg 2013: 17). Am Ende des 18. Jahrhunderts tauchte der Begriff dann häufiger in Medizin, Naturgeschichte, Geographie und Finanzwesen auf. Er bezeichnete hier zumeist - ganz im Sinne Rheinbergers oder Latours - Tatsachen in empirisch durch Experiment, Erfahrung oder Sammlung belegten Beweisen, also eher Resultate als Grundlagen der Empirie. Diese semantische Inversion ermöglichte Daniel Rosenberg zufolge die ubiquitäre Verbreitung des Begriffs in der Ära des Computerzeitalters, in welcher „Daten“ sowohl als Voraussetzung eines Arguments als auch deren Ergebnis, vor allem aber als Information in numerischer Form verstanden und allgegenwärtig wurden. Das 19. Jahrhundert sieht Rosenberg in dieser Entwicklung als „kulturelle Latenzphase“ an, das heißt als eine Zeit, in welcher der Begriff zwar allmählich in weiteren Bereichen Verwendung fand, aber im generellen Sprachgebrauch eine untergeordnete Rolle spielte (Rosenberg 2013: 38).

Mit Blick auf die deutschsprachige Bevölkerungsstatistik bestätigen sich Rosenbergs Befunde insofern, dass numerische Erfassungen in staatlichem 
Auftrag zwar bereits im 18. Jahrhundert vielfach vorkamen (Behrisch 2016), von „Daten“ vor 1860 jedoch nur in wenigen Texten die Rede war. Gleichwohl kam dem Begriff in seiner uns heute geläufigen, numerisch ausgerichteten Konnotation seit Beginn des 19. Jahrhunderts richtungweisende Bedeutung zu. Er wurde von führenden Theoretikern jener Zeit mit Bedacht gewählt, wenn es darum ging, die Statistik als neue Wissenschaft zu etablieren. Der Göttinger Gelehrte August Ludwig von Schlözer (Nachfolger des Begründers der statistischen "Staatswissenschaft" Gottfried Achenwall) verstand die neue Wissenschaft 1804 als "stillstehende Geschichte“ und sah ihren Kern „in der Kunst statistische Data zu erkennen und zu würdigen, solche zu sammeln und zu ordnen." (Schlözer 1804: $\mathbb{2} 25$ ). Der hessische Kameralist Wilhelm Butte schloss sich 1808 Schlözers Diktum an; in seiner Schrift „Statistik als Wissenschaft bearbeitet“ plädierte er dafür, „Data“ zumindest im Sprachgebrauch der Experten zu fixieren und begrifflich deutlich von „Fakta“ zu unterscheiden. Im Gegensatz zum Faktum, das ausschließlich historisch „Erwirktes“ bezeichne und „niemals der Statistik angehören soll,“ sei es allein dem „Datum“ vorbehalten, sich „unmittelbar an den Tatbestand des Seyenden" zu halten und von ursächlichen Zusammenhängen zu abstrahieren. In diesem Unterschied sah Butte die grundlegende Trennung von historisch orientierter Staatsbeschreibung und quantifizierender Statistik, und letztere solle künftig als „akademische Hauptbeschäftigung" betrieben werden (Butte 1808: 248 f.; Ernst 2003: 995-999).

Im preußischen Amtsgebrauch setzte sich eine solch eindeutige Festlegung auf den Begriff "Daten" und dessen Deckung mit Zahlen erst im Zusammenhang mit der Reform der Volkszählung in den 1860er Jahren vollständig durch. ${ }^{5}$ Im dann zu Tage tretenden Bemühen, nicht nur das statistische Erhebungsverfahren nach strengen methodischen Standards neu auszurichten, sondern vor allem auch die Auswertung des Erhobenen völlig neu zu gestalten, kommt dem semantischen Befund der Verwendung des Begriffs „Daten“ besondere Bedeutung zu: Denn sein Gebrauch ging mit genau jenem Prozess der Verdatung einher, das heißt der Entwicklung neuartiger Methoden, Werkzeuge und Techniken zur Gewinnung standardisierter Einzelinformationen und deren variabler Verarbeitung zu statistischem Zahlenmaterial und Aggregaten, die nun eingehender diskutiert werden sollen. 


\section{Verdatung in der Praxis}

Das Bestreben, statistische Erhebungen bei Volkszählungen nicht mehr nur als Kopfzählung oder als „Staatsbeschreibung“ sondern als „Volksbeschreibungen“ im Sinne einer quantitativen, auf Zahlen basierenden Dokumentation und Analyse der Zusammenhänge sozialer und wirtschaftlicher Verhältnisse des Staates zu verstehen und vor allem durchzuführen, setzte sich in Europa zwischen 1830 und 1850 unter dem Eindruck der Folgen der Industrialisierung durch (Porter 2003: 28-31). ${ }^{6}$ Es führte in den 1840 er und 50er Jahren in Belgien, Frankreich, Schweden, Großbritannien und den USA, wie auch in Österreich oder dem Königreich Sachsen zu wesentlichen Erweiterungen und Standardisierungen der Erhebungskriterien, die vor allem im Hinblick auf die politischen und sozialen Implikationen der statistischen Kategorisierung historisch aufgearbeitet worden sind. ${ }^{7}$ Die preußische Volkszählung blieb, obgleich schon früh institutionell im Königlich-Preußischen Statistischen Bureau verankert, bis 1860 einem System des frühen 19. Jahrhunderts verhaftet. ${ }^{8}$ Dies änderte sich 1860 mit der Berufung von Ernst Engel, einem Bergbauingenieur der sächsischen Bergakademie in Freiberg, zum neuen Direktor des Königlich-Preußischen Statistischen Bureaus. Engel, der vormals Leiter des sächsischen Zensusbüros gewesen war, stand in enger Verbindung mit führenden Statistikern Europas: Er hatte ein prägendes Jahr bei Frederic LePlay in Paris verbracht, Quetelet in Belgien aufgesucht und war Mitbegründer des 1853 ins Leben getretenen Internationalen Statistischen Kongresses. ${ }^{9}$ Auf den Tagungen dieser Vereinigung ging es darum, verbindliche Standards für bevölkerungsstatistische Erhebungen zu erarbeiten und für deren Durchsetzung auf nationaler Ebene zu sorgen. ${ }^{10}$ Die grundsätzliche Reform des preußischen Zensus, die Engel gleich nach seinem Amtsantritt anstieß, basierte auf diesem europäischen Diskurs und auf Erfahrungswissen aus eigener Anschauung, das der preußische Direktor sich etwa durch Besuche bei seinen Amtskollegen in Brüssel und London verschaffte (Schneider 2013: 253), oder auf den Versammlungen des internationalen statistischen Kongresses sammelte. So kam es in Preußen zu einer Kombination von best practices für Erhebung und Kompilation, von denen sich einige in ähnlicher Form bereits in anderen Ländern bewährt hatten.

Engels Beitrag, die Bevölkerungsstatistik in Preußen als empirisch begründete Verwaltungspraxis zu etablieren, um strukturelle Probleme der sich schnell wandelnden Gesellschaft freizulegen, sind heute unstrittig, auch wenn dem langjährigen Direktor des Statistischen Bureaus aufgrund seiner begrenzten mathematischen Fähigkeiten vor allem unter Wissenschaftshistorikern bisweilen ein wenig schmeichelhafter Ruf anhaftet. Insbesondere Ian Hackings Befund, Preußens Statistisches Bureau habe unter 
Engels Leitung eine „moraine of cluttered numbers“ ohne jedes Konzept für statistische Gesetzmäßigkeiten produziert, hat im angelsächsischen Kontext dazu beigetragen, die preußische Statistik des 19. Jahrhunderts gegenüber der französischen statistischen Probabilistik insgesamt als wenig bedeutsam anzusehen. ${ }^{11}$

Weit weniger bekannt ist hingegen Engels Rolle als Erneuerer der methodischen und technischen Modalitäten einer wissenschaftlichen Standards folgenden Bevölkerungsstatistik, die erst jüngst eine ausführliche Darstellung erfahren hat (Schneider 2013: insb. 223-282). Diese gibt Aufschluss über die wichtigsten konzeptionellen und technischen Neuerungen im preußischen Zensus, auf die ich im folgenden zurückgreife; auch bietet sie wertvolle Einblicke in die politischen Rahmenbedingungen der Reform und die Auseinandersetzungen Engels innerhalb wie auch außerhalb der preußischen Regierung. Fragen der materiellen Kultur und der konkreten Praktiken bei der Verarbeitung der Erhebungen, wie sie im Rahmen dieses Beitrags interessieren, werden jedoch nur zum Teil berührt und nicht eingehend analysiert; dadurch bleibt die epistemische Bedeutung der Reform insgesamt unterschätzt. ${ }^{12}$

Die im Hinblick auf die Verdatung zentrale, konzeptionelle Umwandlung des preußischen Zensus beinhaltete mehrere Schritte. Die erste, grundlegende Neuerung, die Engel in Anlehnung an die Empfehlungen der internationalen statistischen Kongresse einführte, war eine strenge methodische Unterscheidung zwischen dem erhobenen „Urmaterial“ auf der einen und der resultierenden "Tabelle“ statistischer Ergebnisse auf der anderen Seite. In seiner 1861 erschienenen wegweisenden Abhandlung über die Methoden der Volkszählung führte er dies folgendermaßen aus: „Wir unterscheiden streng zwischen Liste und Tabelle. Erstere bezieht sich stets auf eine Species, auf das einzelne Individuum. In Letzterer ist die Species, das Individuum, nicht mehr erkennbar; sie enthält schon ein concentrirtes Resultat, eine Zusammenfassung und Gruppierungen der Angaben aus den Listen“" (Engel 1861: 163).

Die Bündelung von Einzeldaten zu verschiedenen Aggregaten war bis dahin in der preußischen Statistik nicht erfolgt, weil nicht zwischen Liste und Tabelle unterschieden worden war. Engel führte dies als einen der entscheidenden Gründe für die mangelnde methodische Fortentwicklung der preußischen Statistik an. Die statistischen Erhebungen erschienen seit 1807 im Druck genau so wie das Material gesammelt worden war: Als inzwischen buchdicke Liste mit 625 oder mehr Rubriken aller gezählten Einheiten, eine Darstellung, die Engel nicht nur epistemologisch unbefriedigend, sondern auch ästhetisch als beleidigend empfand (Engel 1861: 163; hierzu auch Schneider 2013: 228). Selbst weit entfernt davon, ein Aufhäufen von Einzelheiten zu verfolgen, warf Engel seinen Vorgängern vor, staat- 
liche Statistik ohne Blick für das Wesentliche in einem starren, additiven Spaltenkorsett betrieben zu haben: Es handele sich bei statistischer Arbeit, so Engel, aber „nicht so sehr um viele, als vielmehr um gute Nachrichten, d.h. also um eine methodische Erhebung, Sammlung und Nutzbarmachung der staatlich wichtigen und interessanten Zustände" (Engel 1861: 156). Zentral für eine solche Zusammensicht sei die Bevölkerungsstatistik, die - verbunden mit einer umfassenden Berufs- und Gewerbestatistik sowohl die wirtschaftlichen als auch die sozialen Strukturen des Staates in ihren gegenseitigen Bedingtheiten sichtbar werden lasse. Ein solch zentraler Status sei der Volkszählung bislang in Preußen aber nicht eingeräumt worden. ${ }^{13}$ Die Unterscheidung von Liste auf der einen und Tabelle auf der anderen Seite galt gleichsam als Gewährleistung einer Reform, in welcher der Volkszählung die ihr gebührende zentrale Stellung zukam.

Als zweite grundlegende Neuerung ersetzte Engel ältere Zählkategorien wie Ortschaften, Gebäude, oder den Haushalt und erhob statt dessen das Individuum zur Grundeinheit des preußischen Zensus, eine Neuerung, die in den 1850er Jahren in etlichen anderen Ländern eingeführt und auf mehreren Tagungen des Internationalen Statistischen Kongresses diskutiert worden war (Engel 1879: 374). Individual- anstelle von Haushaltslisten kamen in Preußen im Jahr 1867 erstmals zum Einsatz. Dies hieß nicht, dass die Einwohner Preußens fortan ungeachtet ihrer Position in Haushalt oder Familie erfasst wurden, im Gegenteil: Nach wie vor gab es für jeden Haushalt eine Liste, die alle Mitglieder umfasste. Ob aber eine Person Ehefrau, Haushaltsvorstand oder nichtverwandter Bediensteter in einer häuslichen Einheit war, wurde nun auch als ein Charakteristikum jedes Individuums erhoben, eine Methode, die nach damaliger Einschätzung eine genauere Analyse von Haushaltsstrukturen zu erlauben schien als zuvor. ${ }^{14}$

\section{Medien der Verdatung}

Die Einführung der Individuallisten ging Hand in Hand mit einem neuen System der Auswertung des Erhebungsmaterials. Besonders aufschlussreich ist, dass der Reformer bei seinen theoretischen Überlegungen zu dieser dritten Neuerung konsequent den Begriff „Daten“ verwandte. Engel führte aus: „Statistik wird durch Methoden definiert. Diese Methoden unterscheiden sich, je nachdem, ob sie auf die Erhebung, Sammlung oder die Nutzbarmachung der Daten abzielen" (Engel 1861: 162). In seinem methodischen Dreischritt der Datenerhebung und -verarbeitung gebrauchte Engel den Begriff um zu beschreiben, dass die einzelnen Angaben im Rohoder Urmaterial Voraussetzungen für Ergebnisse waren und als solche in 
standardisierten Erhebungsbögen erfasst, dann aber nicht nur gesammelt, sondern auch „verarbeitet“, das heißt sortiert und nach verschiedenen Kriterien miteinander kombiniert, in Zahlen zusammengefasst, in Tabellen dargestellt und begleitenden Texten interpretiert und schließlich in Graphiken und Karten visualisiert werden mussten.

Technisch wurde die Umsetzung dieses methodischen Dreischritts mit einem eigens für diesen Zweck entworfenen, losen Papierformular erreicht. Die sogenannten Zählblättchen fungierten als bewegliche Datenträger, die man, sobald die entsprechenden Informationen aus den Erhebungslisten auf diese übertragen waren, nach verschiedenen Kriterien beliebig kombinieren, schnell sortieren und dann auszählen konnte. Sie waren - unter der Bezeichnung cartoline - beim ersten nationalen Zensus des vereinigten italienischen Königreichs im Jahr 1861 verwandt worden, um die vielen regionalen und lokalen Erhebungsverfahren rigoros zu vereinheitlichen und dabei möglichst zügig "dati“ der Bevölkerung des neuen Staates zu erzeugen, welche nationale Identität stiften, aber auch aktuellen statistischen Standards entsprechen und international vergleichbar sein sollten. ${ }^{15}$ Engel lernte die Sortiermethode mit Zählblättchen bei einer „exquisiten Vorführung" auf dem Internationalen statistischen Kongress 1867 in Florenz kennen und war von dieser Methode so angetan, dass er die Blättchen schon im Dezember des gleichen Jahres gemeinsam mit den Individuallisten zur Kompilierung der preußischen Volkszählung erstmals zum Einsatz brachte (Engel 1867: 304).

Bewegliche Papierwerkzeuge, die das Sortieren und Auffinden von Informationen erleichterten, waren seit dem 18. Jahrhundert vor allem im Bibliothekswesen bekannt, aber auch Naturforscher und Humanisten benutzten Zettelkästen, um ihre Aufzeichnungen flexibel $\mathrm{zu}$ ordnen und $\mathrm{zu}$ archivieren (Zedelmaier 2015; Charmentier \& Müller-Wille 2014; Blair 2010; Heesen 2006; Meinel 1995). Die Zählblättchen, die in der Aufbereitungsarbeit des Zensus zum Einsatz kamen, waren allerdings keine gradlinige Fortsetzung dieser Techniken; vielmehr waren sie vom Kartenspielen bzw. Spielkarten inspiriert, die wiederum ebenfalls bibliographischen und archivarischen Papierwerkzeugen zugrunde lagen (Krajewski \& Krapp 2011: 33). Zählblättchen sollten, wie Engel erklärte, aus widerstandsfähigem, geleimtem Papier bestehen und die Größe einer Spielkarte haben (Engel 1867: $303 \mathrm{f}$.$) .$

Engels von Italien übernommene Zählblättchen hatten mehr mit Spielkarten gemein als ihre Größe. Auch wie die Blättchen im Auszählungsprozess zum Einsatz kamen, glich in vieler Hinsicht dem Kartenlegespiel. Die Größe der Blättchen war entscheidend für das flinke Sortieren, ein Vorgang, bei dem nach zuvor festgelegten Kriterien verschiedene Häufchen gebildet werden mussten. Weil auf die begrenzte Oberfläche der Blättchen 
jeweils nur eine Auswahl von Kriterien von den Erhebungslisten übertragen werden konnte, wurden für die verschiedenen Auszählungen jeweils eigene Sätze von Blättchen vorgedruckt, und grundlegende Merkmale wie männlich und weiblich durch die Farbe der Blättchen kenntlich gemacht. Die individuellen, beweglichen Zählblättchen revolutionierten die Arbeit der Auszählung und das Erstellen von Tabellen in der Bevölkerungsstatistik (Rauchberg 1890). Denn bis dahin war die Auswertung von Erhebungslisten des Zensus mit der Methode des Ausstrichelns vorgenommen worden, ein Verfahren, das nicht nur zeitaufwendig und fehleranfällig, sondern auch extrem schwerfällig war und kaum zuließ, mehrfache Kombinationen von Kategorien zu bilden. Die umfangreichste Auszählung nach diesem Verfahren fand 1880 beim achten US-Zensus statt; sie dauerte mehr als acht Jahre an und bewog das US Census Bureau dazu, den Ingenieur Hermann Hollerith mit der Entwicklung einer maschinellen Lösung für den nächsten Zensus zu beauftragen (Campbell-Kelly \& Aspray 2004: 20-26).

In Preußen wurden die beweglichen Zählblättchen zur gepriesenen Technologie der manuellen Verarbeitung von Massendaten - aus genau den Gründen, die eine Generation später als Vorzüge der auf demselben Prinzip basierenden Lochkarte ins Feld geführt wurden: Sie lösten die Einzeleinträge aus dem starren Korsett der Erhebungslisten und brachten die Daten in Bewegung; hierdurch erlaubten sie, unterschiedliche Charakteristika in größerer Zahl zu kombinieren und ermöglichten so ungleich höhere statistische Komplexität, die wiederum neue Einsichten in die Bevölkerungsstruktur und deren soziale Zusammensetzung gestattete. Ganz in diesem Sinne konstatierte Engel im Jahr 1868: „Ist das ja eben der Vorzug der Methode der Zählblättchen, dass sie unzählige Combinationen der einzelnen Daten des Inhalts der Blättchen gestattet" (Engel 1868: 198). Der Hauptunterschied zu der späteren Lochkarte war, dass die Information von den Erhebungslisten auf die Blättchen per Stift übertragen und nicht eingelocht wurden. Und während die Lochkarten dann von Maschinen sortiert und gezählt wurden, musste man die Zählblättchen per Hand in Stapel sortieren und dann auszählen. ${ }^{16}$

Die Zählblättchenmethode, die Engel 1867 in den preußischen Zensus einführte, verbreitete sich in den folgenden Jahren schnell und fand mit leichten Abweichungen vom preußischen Modell - in anderen deutschen und etlichen europäischen Staaten Anwendung (Mayr 1914: 123). Die bayerische Variante, die 1871 erstmals zum Einsatz kam und in acht Farben zur Markierung von Geschlecht und jeweiligem Zivilstand ausgegeben wurde, übernahm die britische Kolonialmacht für den indischen Zensus von 1901 und 1911. Sie erweiterte hierbei das bayerische System auf zwei verschiedenfarbige Sätze von insgesamt 15 Zählblättchen, deren Ecken in verschiedener Weise abgeschnitten waren, um über Geschlecht 
und Zivilstand hinaus Kriterien wie Ethnizität, Kaste und Konfession über die Form der Blättchen kenntlich zu machen (Mayr 1902). In Europa blieb das britische General Register Office der alten Strichelmethode am längsten verhaftet, was zur Folge hatte, dass der britische Zensus sich bis zur Einführung des Lochkartensystems im Jahr 1911 auf die Erhebung weniger Kriterien beschränkte und die Statistiken kaum Kombinationen von Variablen enthielten (Higgs 2004: $213 \mathrm{f}$.).

Wie sehr Zählblättchen die amtliche Bevölkerungsstatistik tatsächlich veränderten, bewies Hermann Schwabe, der Direktor der statistischen Kommission der Stadt Berlin, im Jahr 1869. Schwabe setzte hierfür die Zählblättchen zur Auswertung der Berliner Materials in einer Art und Weise ein, die weit über das Mandat der preußischen Volkszählung hinausging, um eine differenzierte Analyse der Berliner Bevölkerungs- und Berufsstruktur, wie auch der Wohnverhältnisse der preußischen (und später reichsdeutschen) Hauptstadt vorzulegen (Schwabe 1869). Die neuen Papierwerkzeuge erlaubten es Schwabe, Tabellen zu produzieren, welche die Altersstruktur der Berliner nach Geschlecht, Alter, Zivilstand und sozialer Stellung darstellten, wozu allein auf der x-Achse vier Variablen nötig waren. Die Variablen von Alter, Geschlecht und Zivilstand wurden zusätzlich in Kombination mit selbständig und unselbständig Beschäftigten und ihren abhängigen Familienangehörigen dargestellt. Ebenso komplex war eine Tabelle, welche die Wohnstrukturen der beiden Berufsgruppen nach Geschlecht getrennt aufzeigte: $\mathrm{Ob}$ sie mit ihrem Arbeitgeber oder in ihren eigenen vier Wänden lebten, oder als sogenannte Schlafgänger, die sich nur ein tagsüber ungenutztes Bett leisten konnten. Eine Reihe weiterer breit aufgegliederter Tabellen klärte über die Wohndichte in Berlins Mietshäusern auf, unterschieden nach Vorder- und Hinterhäusern, wie auch Kellerbis Dachwohnungen. Die Aufstellungen machten nur allzu deutlich, wie absichtsvoll die neue Technik dazu genutzt wurde, um brennende soziale und politische Fragen der Zeit statistisch in den Blick zu nehmen. ${ }^{17}$

Zusätzlich bestückte Schwabe seine Studie mit insgesamt 24 farbig gestalteten, sorgfältig aufbereiteten graphischen Darstellungen der statistischen Ergebnisse. Sichtlich beeinflusst von den internationalen Debatten über Gebrauch und Nutzen graphischer Darstellungsformen in der Statistik, an denen er selbst aktiv beteiligt war, setzte Schwabe mit seinem Werk Standards. Er demonstrierte, dass die neuen technischen Methoden die Bevölkerungsstatistik zu einer Wissenschaft machten, die nicht nur detaillierte Zahlen und komplexe Tabellen zu produzieren im Stande war, sondern auch neue graphische Darstellungen ermöglichte, mit denen die Resultate der Erhebungen effektiv kommuniziert werden konnten. ${ }^{18}$ Für Engel diente Schwabes bahnbrechende Studie als willkommener Beleg dafür, was die von ihm in Preußen eingeführte neue Technik zu leisten im Stande 
war. Seiner Meinung nach verdankte sich die Vielschichtigkeit von Tabellen und Graphiken einzig und allein der neuen Methode der Datenverarbeitung per Zählblättchen, die dadurch „nicht nur technische, sondern auch moralische und pädagogische Bedeutung erlangt" (Engel 1870: 39).

Um die Auswertung des Erhebungsmaterials noch effizienter zu gestalten, führte Engel für den Zensus von 1871 die sogenannte Individual-Zählkarte ein, eine dünne Pappkarte, die ungefähr viermal so groß war wie ein Zählblättchen und allen Angaben Platz bot, die von jeder Person abgefragt wurden. ${ }^{19}$ Die Zählkarte stellte eine Verschmelzung von Erhebungsliste und Zählblättchen dar, um den aufwendigen Prozess der Datenübertragung von einem Medium auf das andere obsolet zu machen. So diente die Zählkarte zunächst als Erhebungsformular, das in jeden Haushalt verschickt wurde, um hier auch möglichst von den Gezählten selbst ausgefüllt zu werden. ${ }^{20}$ Die ausgefüllten Karten wurden dann eingesammelt und geprüft, aber ohne zusätzlichen Übertragungsvorgang genauso genutzt wie zuvor die Zählblättchen. Einmal auf ihre Richtigkeit kontrolliert, konnten sie sogleich in Stapel sortiert, verschieden kombiniert und ausgezählt werden, bevor die ermittelten Zahlen in Zwischentabellen vermerkt, geprüft, zusammengezogen und schließlich gedruckt wurden.

Mit der Einführung der Zählkarte im Jahr 1871 hatte das preußische statistische Büro ein System der Verarbeitung von Massendaten etabliert, mit dem sich Bevölkerungstabellen in einer Schnelligkeit, Qualität und Differenzierung von Kombinationen produzieren ließen, die, wie Engel stolz betonte, „bisher auch nicht annähernd erreicht wurde und wie kein anderes Land der Welt dem etwas ähnliches an die Seite zu setzen hat.“21 Nicht alle Statistiker schlossen sich dieser Meinung an; sie favorisierten die Kombination von Erhebungsliste und Zählblättchen, eine Variante, die zum Standard des Auszählungsverfahrens wurde (Mayr 1893). In Preußen jedoch trieben Nationalstolz und Vormachtstreben die statistische Innovation per Zählkarte mit der Reichsgründung Deutschlands weiter voran.

\section{Logistik, Zirkulation, Arbeit}

Die preußische Volkszählung von 1871 war die erste, die mit mithilfe von Individual-Zählkarten erhoben und ausgewertet wurde. Gleichzeitig war es auch der erste Zensus, bei dem das Statistische Bureau die gesamte Ausbeutung des Materials zentralisierte und in Berlin vornahm (Schneider 2013: 259). Zu diesem Zweck musste die Behörde Arbeitsabläufe der Datenverarbeitung schaffen, die von knapper Budgetierung, rigiden Zeitplänen und hohen Erwartungen an die Qualität der Ergebnisse diktiert 
wurden. Um diesen Herausforderungen begegnen zu können, schuf man ein System der zirkulären und überwiegend auf Stücklohn basierenden Auswertung, das sich über ganz Berlin erstreckte. Eine Rekonstruktion dieses Systems erlaubt genaue Einblicke, was mit dem Erhebungsmaterial geschehen musste, bis man die Tabellen in den neuen Veröffentlichungen des Bureaus studieren konnte. Diese Praktiken bringen zum Vorschein, wie sehr die Materialität der Daten den Prozess ihrer Verarbeitung diktierte.

Als die 5.000 Kisten mit insgesamt 375 Tonnen Gewicht und 29 Millionen ausgefüllter Zählkarten aus allen Zähldistrikten Preußens im Dezember 1871 in Berlin eintrafen, heuerte das Bureau 300 zusätzliche Arbeiter an, um die eingetroffenen Karten zu überprüfen und das Sortieren und Zählen der Karten zu bewerkstelligen (Engel 1873b: 356). Das Gebäude des Bureaus war 1869 neu errichtet worden, aber auf die zentrale Auswertung des preußischen Zensusmaterials nicht ausgelegt. Fehlender Lagerplatz erforderte ein ständiges Bewegen der Kisten innerhalb des gesamten Gebäudes und schuf ein lautes, lähmendes Durcheinander; auch erforderte das Sortieren der Karten auf Häufchen deutlich mehr Platz als gedacht (Blenck 1905: 266). Der ursprüngliche Plan einer zentralisierten Ausbeutung im Amt selbst wurde daher schnell aufgegeben, und die eigentliche Arbeit der Aufarbeitung fand schließlich größtenteils nicht im Bureau selbst statt, sondern wurde als Heimarbeit vergeben.

Die Beschäftigung von Frauen für manuelles computing ist in der historischen Forschung nicht unbekannt und in Einzelfällen auch als Arbeit überliefert, die zu Hause durchgeführt wurde. ${ }^{22}$ Im Zuge der Volkszählungsarbeiten für das Preußische Statistische Bureau nahm die weibliche Heimarbeit ab 1871 allerdings erstaunliche Ausmaße an. Die Zirkulation der Karten zwischen dem Bureau und zahlreichen Wohnungen Berlins wurde zum zentralen Bestandteil der manuellen Datenverarbeitung, und der Stücklohn nahm von Jahr zu Jahr zu. Ein Höhepunkt war 1895 erreicht, als Engels Nachfolger Emil Blenck im Rahmen der Berufszählung 1.000 Arbeiter in Zeitlohn und ca. 3.000 Stücklohnarbeiter in Heimarbeit für die Auszählung der umfangreichsten Erhebung beschäftigte, die das Bureau jemals unternommen hatte. Zwischen Oktober 1895 und Mai 1896 wurden 27.760 Kisten mit Arbeitsmaterial von je etwa anderthalb Zentner Gewicht kreuz und quer durch die Stadt bewegt; $2.137 .600 \mathrm{~kg}$ Erhebungsmaterial wollten dabei bewältigt werden. Über ganz Berlin und seine Vororte war zeitweilig das Zählmaterial verstreut (Blenck 1897: 204). Nur wenige fest bestallte Beamte dirigierten ein Heer von Hilfsarbeitern samt ihren Familien und wachten über die Masse an „ununterbrochen im Kreislauf sich bewegenden Zählpapieren“ (Blenck 1897: 302). Jede Kiste ging bis zu drei Mal zwischen dem Amt und einem Haushalt hin und her. Jede Zählkarte wurde bei dieser Prozedur drei großen Zählungen unter- 
worfen und dabei viele Male nach unterschiedlichen Kriterien neu sortiert und gezählt, bis alle für die neuen Bevölkerungstabellen erforderlichen Zahlen in allen verlangten Kombinationen ermittelt waren (Blenck 1892: 214). Die ausgreifende Maschinerie der manuellen Datenverarbeitung verwandelte Berlin in ein feinmaschiges Netz aus Akteuren, Anlaufpunkten und Fahrstrecken, dessen Bewegung einer von den Datenträgern und ihren häuslichen Bearbeiterinnen bestimmten Choreographie folgte, die nur wenige zu steuern wussten. Dies galt insbesondere für die Organisation der Zustellung und Abholung des zur Heimarbeit bestimmten Materials. Diese Aufgabe oblag einem langjährigen Hilfsarbeiter des Bureaus, der nicht nur über ein geographisches Verständnis der einzelnen Zählbezirke Preußens verfügte, sondern offenbar auch ein gleichsam bibliothekarisches Gedächtnis darüber besaß, welche Kiste sich zu welcher Zeit in welchem Haushalt befand, und wo sie als nächstes sein musste. ${ }^{23}$

Die stetige Zunahme an Vergabe von Heimarbeit geschah nicht nur aus Platzmangel im Statistischen Bureau, sondern kam dem Auszählungsprozess als solchem entgegen. Die über Jahrzehnte anhaltende Ausweitung von Heimarbeit an die meist weiblichen Angehörigen der im Bureau selbst beschäftigten Hilfskräfte verweist darauf, dass die Datenverarbeitung nach preußischer Art sich auf eine ganz bestimmte geübte, eingespielte und dennoch flexibel abrufbare Belegschaft angewiesen sah. Die überlieferten Quellen belegen, wie sorgfältig die Direktoren des Bureaus ihre Hilfsarbeiterschaft über Jahrzehnte aufbauten. Die Mehrzahl der langfristig auf Tageslohnbasis beschäftigten männlichen Hilfsarbeiter waren nicht mehr ganz junge Männer, die aus früheren Beschäftigungen über Erfahrung in Buchhaltung, Bankwesen, Versicherung oder Schreibarbeit verfügten; auch gab es ehemalige Kaufleute, pensionierte Gymnasiallehrer oder Landwirte unter ihnen. Alle waren verheiratet. ${ }^{24}$ Während die männlichen Hilfskräfte Auszählungsarbeiten im Bureau selbst verrichteten, führten ihre Ehefrauen und weiteren Verwandten dieselbe oder ähnliche Arbeit daheim aus. Wann immer der Bedarf an Arbeitskräften die Kapazitäten im Bureau selbst überstieg, rekrutierten die Beamten zuerst die Angehörigen ihrer Hilfsarbeiter. Erst wenn auch diese Reserve ausgeschöpft war, annoncierte die Behörde öffentlich, um neue Kräfte und vor allem Veteranen anzuwerben, die einen Anspruch darauf hatten, vorrangig für derlei Aufgaben im Bureau beschäftigt zu werden. Sehr zum Ärger der Letzteren ging das Bureau jedoch gleichzeitig dazu über, die häusliche Auszählung mehr und mehr nicht nur als zusätzlichen Stücklohnverdienst für seine Hilfsarbeiter anzubieten. In Hochphasen schloss die Rekrutierung häuslicher Mitarbeit bald auch die Familien der mittleren Beamten und Sekretäre ein. Während der Berufszählung von 1895 etwa setzte das Bureau explizit Verbote aus, den Angehörigen fest bestallter Beamten Heimarbeit anzuvertrauen. 
In der Folge glichen manche Beamtenhaushalte bald geschäftigen Sortierbüros. So versorgte einer dieser Aufträge neben der Ehefrau des Sekretärs acht weitere Menschen mit Einkommen: Zwei Schwägerinnen und den Schwager des Hausherrn, zwei Witwen und zwei unverheiratete Frauen aus der Nachbarschaft sowie einen arbeitslosen Kaufmann. Die Arbeit dieser Gruppe wurde als so exzellent erachtet, dass das Bureau ausdrücklich die erlaubten Höchstgrenzen für zusätzliches Einkommen aussetzte. Als Begründung hierfür führte die Behörde an, dass die Hausfrau eine Dienstbotin habe einstellen müssen, um sich selbst ganz den Auszählungsarbeiten widmen zu können. Arrangements wie dieses waren kein Einzelfall. Denn die häuslichen Auszählteams waren weder an den Achtstundentag im Amt noch an dessen Sonn- oder Feiertagsruhe gebunden und mitunter über Wochen täglich bis zu sechzehn Stunden am Werk. ${ }^{25}$

Kontrolle wurde grundsätzlich durch die männlichen Haushaltsvorstände ausgeübt, die nicht nur für ihre eigenen Arbeiten, sondern auch für die ihrer Angehörigen bzw. zu Hause mit Arbeitenden verantwortlich waren und bezahlt wurden. Zusätzlich überprüften Revisoren im Bureau die Ergebnisse nach jeder Auszählungsrunde. Zu besonderen Stoßzeiten fuhren Prüfer von einem Haushalt zum nächsten, um die Zeit des Hin- und Hertransports der Kisten zum Bureau einzusparen. Schlampige, fehlerhafte Arbeit wurde mit empfindlichen Lohneinbußen geahndet, um Korrekturen und Zweitauszählungen auf ein Minimum zu beschränken. Letztere wurden zudem auf Kosten der Verursacher durchgeführt. Der Einkommensverlust wegen unzulänglicher Ergebnisse konnte bis zu 84 Prozent des Lohns betragen und belief sich im Durchschnitt auf zehn Prozent. ${ }^{26}$

Die soziale Verortung der Zustellungen des Bureaus - soweit sie sich rekonstruieren lässt - verweist darauf, dass die Statistiker vor allem Haushalte im kleinbürgerlichen Milieu Berlins im Auge hatten, derjenigen Schicht, die Kaiser, König und Staat im Gegensatz zur Arbeiterschaft besondere Loyalität entgegenbrachte (Oertzen 2018). Insbesondere von den hier waltenden Ehefrauen erwarteten die Beamten, eine wie es hieß „geordnete Häuslichkeit" zur Verfügung zu haben, der man das wertvolle Urmaterial zur Auszählung getrost anvertrauen durfte. ${ }^{27}$ Die amtliche Erwartung an den ordentlichen Haushalt bezog sich auf eine konkrete Räumlichkeit: denn es war die meist ungenutzte, aber peinlich sauber gehaltene "gute Stube", wo die Auszählung in Heimarbeit von statten zu gehen hatte. An diesem mit dem nötigen Platz und einem ausreichend großen Tisch versehenen Ort führten die Frauen einen komplexen Auftrag aus, nachdem sie wie alle anderen Hilfsarbeiter über zwei bis drei Tage im Statistischen Bureau in die Arbeit eingewiesen worden waren. ${ }^{28}$

Eine nähere Betrachtung der einzelnen Arbeitsschritte verdeutlicht, dass die Heimarbeit als Routine, aber keinesfalls als mechanischer Automatis- 
mus zu betrachten ist. Die Frauen hatten das in der Kiste verstaute Urmaterial daheim in Empfang zu nehmen; sie mussten die Kiste vorsichtig auspacken, das Material auf Vollständigkeit prüfen und dann mit der Sortierung beginnen, wobei der beigegebene Legeplan auf das Strengste zu befolgen war. Denn von der Ordnung der Karten hing über den gesamten Verarbeitungsprozess alles ab; jeder weitere Sortiervorgang baute auf dem Zustand auf, in dem die Zählkarten sich zuvor befanden. Lagen alle Karten auf den richtigen Häufchen, hatten die Heimarbeiterinnen jedes durchzuzählen, die sich ergebenden Zahlen mit schwarzer Tinte in die jeweiligen Spalten des Tabellenformulars einzutragen, und Zwischen-, Gesamt- und Kontrollsummen zu errechnen. Ergaben sich Fehler, waren diese aufzuspüren und in Rot zu korrigieren. Schließlich musste jedes Häufchen in seiner neuen Ordnung in Papier verpackt, beschriftet und verschnürt zurück in die Kiste gelegt werden, damit diese zur Zwischenkontrolle zurück ins Bureau verbracht werden konnte (Blenck 1892: 214 f.).

Loyalität, Ordentlichkeit, Sorgfalt, Ausdauer und Sicherheit im Rechnen waren nicht die einzigen Fähigkeiten, die den Heimarbeiterinnen abverlangt wurde. Das Layout der Zählkarten erzwang, die meisten Einträge als Worte in vorgegebene Antwortfelder auf gepunktete Linien zu schreiben. Edward Higgs hat am Beispiel des britischen Zensus gezeigt, wie schwierig es schon beim Ausfüllen der Formulare war die richtigen Angaben etwa darüber zu machen, wie viele "Räume“ und Angehörige ein Haushalt umfasste, je nachdem was man unter diesen Begriffen verstand (Higgs 1996: 71). Wieviel Interpretation nach der Erhebung ganz abgesehen von der Entzifferung millionenfach verschiedener Handschriften vonnöten war, um alle Angaben auf den Zählkarten in die vorgesehenen Spalten einer Tabelle zu subsumieren, wird an dem Hilfsmaterial deutlich, das den Auszählerinnen vom Statistischen Bureau mitgeliefert wurde, etwa zu Frage 2 auf der Zählkarte des Zensus von 1890. Erfordert war hier die auf den ersten Blick simpel erscheinende Angabe, in welchem verwandtschaftlichen oder rechtlichen Verhältnis die betreffende Person zum Haushaltsvorstand stand. Die Tabelle sah acht verschiedene Rubriken vor, um von leiblichen Kindern über Untermieter, Gesinde und einquartierte Soldaten die verschiedensten Formen des Zusammenlebens in eine Übersicht zu bündeln. Eine Hilfstabelle, in der entsprechende Begriffe zur Orientierung bereits eingruppiert waren, listete über 50 verschiedene Bezeichnungen auf - von Mamsell über Aftermieter bis zu Mündel oder Kämmerer -, die den Bearbeiterinnen helfen sollten, jede Karte auf das richtige der acht Häufchen zu sortieren (Blenck 1892: 220). Die Hilfstabelle vermittelt einen Eindruck von den schier unzähligen und nach Landstrichen und Dialekten sich unterscheidenden Möglichkeiten, Frage zwei mit einem Wort zu beantworten, und wie schwierig es sein konnte, jede Antwort richtig zu klassifizieren. Die 
im Design der Zählkarte und in der Methode der Datenerhebung gründenden Schwierigkeiten der Entzifferung und Zuordnung stellten hohe Anforderungen an die Auszähler und Auszählerinnen und machten Erfahrung so wertvoll, dass das Bureau gute Kräfte dauerhaft beschäftigt hielt und deren Angehörige über viele Jahre immer wieder zu Zählungen heranzog (Oertzen 2017).

Das arbeitsteilige System, so fehlerlos und effizient es in den Augen der Statistiker funktionieren mochte, brachte allerdings selbst soziale Konflikte hervor; es verleitete das Bureau, sozialpolitische Regelungen zu umgehen, die es - wie die gesamte preußische Verwaltung - verpflichtete, vorrangig Veteranen mit Hilfsarbeiten zu betrauen. Tatsächlich wurden Veteranen zwar auch eingestellt - doch oftmals als erste entlassen, wenn der Bedarf an Hilfskräften nachließ. Die erhaltenen Akten sind voll von erbitterten Petitionen, in denen die Entlassenen das Statistische Bureau beschuldigten, vor allem Menschen Arbeit zu geben, die „nie gedient haben“ und zudem „das schöne Geschlecht“ zu bevorzugen. ${ }^{29}$ Die Veteranen mutmaßten Günstlingswirtschaft und Korruption am Werk, eine Tendenz, die nicht allein gegenüber dem Statistischen Bureau vorherrschte, sondern den gesamten Staatsdienst betraf, als langgediente Zivilanwärter sich organisierten, um ihren Anspruch auf Beschäftigung einzufordern (Vogel 2001). Konfrontiert mit solcherlei Vorwürfen, verteidigte die Behörde ihre Strategie. Das statistische Bureau seine keine Wohlfahrtseinrichtung, sondern müsse Effizienz an erste Stelle setzen; andernfalls leide nicht nur die Qualität der Ergebnisse, sondern auch die Frist der Fertigstellung, und beides führe zu einer Überziehung des Budgets. ${ }^{30}$

Die Maschinerie der manuellen Datenverarbeitung, die auf Erfahrung, Vertrauen, Kontrolle und ständischem Gehorsam im Dienst der Genauigkeit beruhte, etablierte sich fest in der preußischen Statistik und galt über Preußen hinaus auch zu Beginn der 1890er Jahre als bestmögliches Verfahren der Volkzählung, das die Bevölkerungsstatistik auf neue Grundlagen gestellt hatte. ${ }^{31}$ Die neue Papiertechnologie setzte die Statistiker Dank ihrer sorgfältig ausgesuchten Hilfsarbeiter und deren geübten weiblichen Angehörigen in Stand, die Ergebnisse der Volkszählungen so schnell wie nur irgendwie möglich produzieren, damit die publizierten Tabellen tatsächlich auch die zu statistischen Aggregaten abstrahierten "Gegenwartszustände“ wiedergaben, von denen sich die Statistiker eine wirksame Analyse gesellschaftlicher Problemlagen erhofften.

Die preußischen Statistiker hielten an diesem Verfahren auch dann weiter fest, als das System der amerikanischen Hollerithkarten im neunten USZensus von 1890 erstmals zum Einsatz kam und in den 1890er Jahren die statistischen Büros in Wien, St. Petersburg und Norwegen in fabrikähnliche Maschinensäle verwandelte (Heide 2009). Die preußische Zurückwei- 
sung der Mechanisierung ist jedoch nicht mit behördlicher Schwerfälligkeit, einer generellen Technikfeindlichkeit oder damit zu erklären, dass die Vermarktung der Hollerithmaschinen in Europa nur langsam vorankam (Heide 2008). Vor dem Hintergrund der Methoden, Medien und Praktiken des etablierten manuellen Verfahrens wird vielmehr deutlich, dass die Verdatung der Bevölkerungsstatistik in Preußen längst erfolgt war: durch bewegliche Papierträger und eine aufwändige, streng geregelte Logistik der Zirkulation, die es erlaubte, effiziente Auszählerinnen mit dem manuellen Kompilieren von Daten nach vielen verschiedenen Kriterien zu betrauen. Dass die weiter mechanisierten Prinzipien von Lochkarte, Zähl- und Sortiermaschine die Auswertung erheblich beschleunigten und das Kombinieren von Kriterien weiter erleichterte, war auch in Preußen unbestritten. Methodisch jedoch hielten die Berliner Statistiker ihre manuellen Zählkarten, die zum Einsammeln und gleichzeitig zum Auszählen der Daten genutzt wurden, für weniger fehleranfällig und daher der Lochkarte überlegen, denn auf Letztere mussten die Daten aus Erhebungslisten erst wieder übertragen werden (Versammlung der Polytechnischen Gesellschaft 1896, 1896: 124). ${ }^{32}$ Aus diesem Grund, wie auch aus sozialpolitischem Kalkül setzte das Statistische Bureau in Berlin weiterhin auf die Handarbeit seiner versierten Beamten, Hilfsarbeiter und deren Familien. Die maschinelle Datenverarbeitung mit Lochkarten, Sortier- und Zählmaschinen blieben daher im preußischen Zensus bis nach Ende des Ersten Weltkriegs unerprobt.

\section{Historizität der Verdatung}

Die Verdatung des preußischen Zensus geschah für Engel nicht zum Selbstzweck. Vielmehr sollten die unter hohem Zeitdruck erfassten und verarbeiteten „neuesten Daten“ eine größtmögliche Verbreitung erfahren und nicht nur Grundlage für amtliches Handeln darstellen, sondern den öffentlichen Diskurs befruchten (Blenck 1896: 365), nicht zuletzt, um auch bei der Bevölkerung dafür zu werben, möglichst sorgfältige Angaben zu machen. Engel gestaltete in den 1860er Jahren daher auch die gesamte $\mathrm{Pu}-$ blikationsstrategie des Statistischen Bureaus um; in der neuen Zeitschrift der Behörde sollten die Ergebnisse diskutiert, verglichen und die wichtigsten aktuellen statistischen und staatswissenschaftlichen Fragen erörtert werden. Aufgabe des (nicht jährlich erscheinenden) amtlichen Jahrbuchs hingegen sollte es sein, den "materiellen [...] und sittlichen, geistigen und politischen Culturzustand" des preußischen Staates abzubilden und ein „thunlichst vollständiges Repertorium über den Stand und die Bewegung 
der statistisch erfassbaren Zustände des Staates“ zu bieten, wie Engel 1864 betonte (Preußische Statistik 1864, Rückdeckel). Die „Preußische Statistik“ schließlich erschien in unregelmäßiger Erscheinungsfolge, um die Tabellenwerke des Bureaus allgemein zur Kenntnis bringen (Schneider 2013: 183).

Engel selbst geriet über diese Auslegung der amtlichen Statistik als Wissenschaft, die einer - ihn eingeschlossenen - „kritischen Öffentlichkeit“ (Habermas) bedurfte, immer wieder in Konflikte mit der autokratischen preußischen Regierung, die 1882 schließlich zu seiner Entlassung führten. ${ }^{33}$ Die von ihm geschaffenen Methoden und Praktiken der Datenverarbeitung wie auch die Veröffentlichungsorgane des Bureaus blieben jedoch bestehen und stellten eine wachsende Fülle komplexer Tabellen bereit. Diese bildeten eine in Zahlen und statistische Aggregate abstrahierte Gegenwart ab und erlaubten über die Serialität von Zustandsbeschreibungen zudem eine differenzierte Analyse des historischen Verlaufs wirtschaftlicher und sozialer Entwicklungen in einer Zeit rapiden gesellschaftlichen Wandels. Die in so großer Masse gewonnenen, historisch vergleichbaren Daten wurden Grundlage und Werkzeug der zwischen 1860 und 1890 besonders in Deutschland einflussreichen Historischen Schule der Nationalökonomie. Die Vertreter dieses streng statistisch und empirisch-faktisch ausgerichteten volkswirtschaftlichen Ansatzes standen in enger Verbindung mit bürgerlich-liberalen Verfechtern sozialer Reform; etliche von ihnen hatten zudem engste Verbindung zu Engels Statistischem Bureau durch das von ihm dort 1861 gegründete Statistische Seminar (Schneider 2013: 132-165). Besonders groß war Engels Einfluss auf Gustav Schmoller, Lujo Brentano, Adolf Held und Wilhelm Knapp, deren Anliegen es war, die Ökonomik als praktische Wissenschaft zu etablieren, um Wissen über die zunehmend komplexer werdende Wirklichkeit zu erlangen, Fehlentwicklungen aufzuzeigen und Konzepte zu deren Überwindung vorzulegen (Grimmer-Solem 2003).

Die Verdatung statistischer Erhebungen gab nicht nur der Nationalökonomie des 19. Jahrhunderts neue Impulse. Denn die preußischen Statistiker waren weder die ersten noch die einzigen Experten des 19. Jahrhunderts, die ihr Material als Daten verstanden, diese mit neuen Werkzeugen erfassten, in Bewegung brachten, durch verschiedenste Kombinationen in Beziehung setzten und auf diese Weise neue Erkenntnisse gewannen. Der Gebrauch des Begriffs „Daten“ und die Hinwendung zu datengetriebener Forschung vollzog sich seit Beginn des 19. Jahrhunderts sowohl in der Kameralistik als auch in Bereichen der sogenannten „baconischen“ Wissenschaften, die darauf ausgerichtet waren, aus empirischen Phänomenen Wissen zu gewinnen. So lässt sich in der Taxonomie des ausgehenden 18. und frühen 19. Jahrhunderts die Formierung der Botanik zu einer Informa- 
tionswissenschaft ausmachen: Das Sammeln und Klassifizieren von Arten wurde hier zu einem Forschungsfeld, in dem Taxa und Namen mehr und mehr nur als Infrastruktur dienten, um die Zirkulation neuer Daten zu bewältigen und zu verbessern. Dieses induktive Nutzen von Strukturen, wie auch das Zählen von Spezies führte zu überraschenden Einsichten vorher nie bemerkter Zusammenhänge. Sie erschütterten überlieferte Vorstellungen, die Natur sei unveränderlich und konstant (Müller-Wille 2017). Eine ähnliche Entwicklung lässt sich anhand der quantitativen Techniken ausmachen, mit denen Kameralisten im frühen 19. Jahrhundert Informationen verarbeiteten, um Strukturen und Gesetzmäßigkeiten in verschiedensten Bereichen vom Ackerbau über die Forstwirtschaft bis hin zur Paläontologie aufzudecken (Sepkoski \& Tamborini 2018). Eine Schlüsselrolle im Transfer entsprechender Praktiken nahm hier der Kameralist Georg Heinrich Bronn ein, der vorhandene paläontologische Kompendien und Kataloge neu sortierte, kompilierte, speicherte und die hierdurch sichtbar werdenden Muster in Diagrammen visualisierte. Bronns Papiertechniken funktionierten wie Datenbanken, mit deren Hilfe er erstmals temporale Strukturen des Aussterbens fossiler Arten sichtbar machte (Sepkoski 2017).

Die Verdatung empirischer Forschung im 19. Jahrhundert war Ausdruck einer neuen, innovativen Reflexivität gegenüber kumulativen wissenschaftlichen Methoden, wie sie sich auch in den monumentalen archivarischen Großprojekten jener Zeit offenbarte (Daston 2017). Technisch beruhte sie auf ähnlichen Papiertechnologien, die auch im 17. und 18. Jahrhundert eingesetzt worden waren, um Informationen aufzuzeichnen, zu ordnen und flexibel miteinander in Beziehung zu setzen. Bewegliche Blättchen und Formulare ebenso wie hieraus abgeleitete Ordnungstechniken lösten bestimmte Sets erhobener Einzelheiten aus dem Raster von Listen und gestatteten unterschiedliche Zuordnungen, ohne die Grundstruktur der Zusammenhänge zu zerstören. Datengetriebene wissenschaftliche Tätigkeiten des 19. Jahrhunderts zielten jedoch auf die Erzeugung von Zahlen und quantitativen Größen; sie befeuerten das Denken in zeitlichen Relationen und räumlichen Strukturen, eine Wahrnehmung, die zu überraschenden neuen Einsichten durch Visualisierung führten, sei es in der Form von (komplexen) statistischen Tabellen oder graphischen Darstellungen neuer Art. In ihrem Bemühen, Strukturen sichtbar zu machen, waren solche wissenschaftlichen Aktivitäten keinesfalls frei von Theorien oder Hypothesen. Vielmehr unterwarfen sie ihre Erfassungskriterien wie auch ihr Material langen und mühseligen Prozeduren, in denen sie die in Frage stehenden Daten konstruierten, messbar machten, klassifizierten, aus ihrem ursprünglichen Kontext lösten und vielfachen Sortiervorgängen unterwarfen. Wie das preußische Beispiel zeigt, erforderte gerade dieser Vorgang der Verarbeitung neue, bewegliche Speichermedien, die wiederum strengs- 
te Ordnung, hochverlässliche Arbeitskräfte für die richtige Zuordnung der Daten und eine ausgeklügelte Logistik der Zirkulation verlangten. Die resultierenden Nachschlagewerke, Tabellen, Graphiken, Karten und sonstigen Abbildungen dienten zum einen als Endergebnis wissenschaftlichen Tuns, zum anderen aber stellten sie die Grundlage für neues Wissen dar. Sie führten, wie die hier skizzierten Beispiele zeigen, zu epochalen historischen Einsichten - obgleich die zugrunde liegenden „Daten“ zuvor strikt als "Seiendes“ aufgefasst, aus ihren kausalen und „geschichtlichen“ Bezügen gelöst und dann zu Zahlen aggregiert worden waren. Dieser Historizität der Verdatung haben Forschungen zur Quantifizierung bislang wenig epistemisches Gewicht beigemessen. Der Wissenschaftsgeschichte soll die durch ein Nachdenken über Big Data angeregten genealogischen und praxeologischen Erkundungen des Begriffs „Daten“ und der mit ihm einhergehenden Werkzeuge, Techniken und Verarbeitungsprozesse als Anregung dienen, sich dem Erkenntnispotential quantitativer, induktiv vorgehender „baconischer" Wissenschaften noch intensiver zu widmen, als dies bislang geschehen ist.

\section{Danksagung}

Danken möchte ich Anna Echterhölter, Staffan Müller-Wille, Sybilla Nikolow, Viktoria Tkaczyk und zwei anonymen Gutachtern für ihre wertvollen Kommentare und Anregungen. Laura Selle hat Ordnung in den Anmerkungsapparat und das Literaturverzeichnis gebracht, auch ihr herzlichen Dank.

Funding Open access funding provided by Max Planck Society.

Open Access Dieser Artikel wird unter der Creative Commons Namensnennung 4.0 International Lizenz (http://creativecommons.org/licenses/by/4.0/deed.de) veröffentlicht, welche die Nutzung, Vervielfältigung, Bearbeitung, Verbreitung und Wiedergabe in jeglichem Medium und Format erlaubt, sofern Sie den/die ursprünglichen Autor(en) und die Quelle ordnungsgemäß nennen, einen Link zur Creative Commons Lizenz beifügen und angeben, ob Änderungen vorgenommen wurden.

\section{Anmerkungen}

1 Vgl. Blair (2010); Müller-Wille \& Charmantier (2012); Krämer (2014); Roberts (2011); Daston \& Lunbeck (2011).

2 Vgl. Porter (1995); Hacking (1990); Desrosières (2005); für das späte 18. Jahrhundert Behrisch (2016). 
3 Zur materiellen Kultur von Wissenspraktiken siehe Becker \& Clark (2001); Krajewski (2002); Klein (2003); Heesen (2005, 2006); Yates (2005); Friedrich, Brendecke \& Friedrich (2008); Soll (2009); Hess \& Mendelsohn (2013); Charmentier \& Müller-Wille (2014). Zur historischen Praxeologie siehe insbesondere Haasis \& Rieske (2015). Zu Aufschreibepraktiken von Zensusinformation im 18. Jahrhundert siehe insbesondere Tanter (2007) und Behrisch (2016); für das 19. Jahrhundert vor allem Higgs (2004); zu Praktiken der Erhebung des britischen Zensus und zur Materialität von Volkszählungsformularen und Umfragebögen siehe Brückweh (2015).

4 Zur Genealogie personengebundener Daten im Versicherungswesen siehe Bouk (2017); in den Sozialwissenschaften siehe Lemov (2017).

5 So wies der erste Direktor des Königlich-Preußischen Statistischen Bureaus anlässlich der Gründung der neuen Behörde der staatlichen Statistik noch die Aufgabe zu, „der Wissenschaft mehr historische Data zu verschaffen“ oder, wenn es daran mangele, zumindest Hypothesen zu formulieren, „deren Betrachtung ihm doch einen Fingerzeig gibt, auf welche Gegenstände er seine Aufmerksamkeit zu wenden habe.“ (Krug 1807: IX), siehe hierzu auch Nikolow (1994: 104).

6 Vgl. Porter (2003: 28-30). Zur statistischen Staatsbeschreibung siehe Nikolow (2001); zur Raumvermessung im 18. Jahrhundert siehe Behrisch (2006).

7 Zur sächsischen Bevölkerungsstatistik des 19. Jahrhunderts siehe Schmidt (2005); zum britischen Zensus der Zeit vor allem Higgs (2004); für die USA insbesondere Anderson (1988); Campbell-Kelly \& Aspray (2004); für Frankreich Desrosières (2005); Schweber (2006); zum weiteren historischen Kontext des Aufschwungs der Statistik im 19. Jahrhundert siehe Porter (1995).

8 Dies betraf vor allem Angaben zum Zivilstand, aber auch zur Altersstruktur der Bevölkerung, in der Kinder erst ab fünf Jahren überhaupt erfasst wurden, und die dann Altersstufen in sehr unregelmäßigen Altersgruppen zusammenfasste, die zwar für die militärische Rekrutierung oder ähnliches relevant sein mochten (7-14;14-16;16-19;19-24 etc.), aber keine Vergleichbarkeit zu anderen Staaten zuließen und zudem weder Aussagen zur Kindersterblichkeit ermöglichten noch die Erstellung von Mortalitätstabellen. Vgl. Engel (1861: 156-58).

9 Zu Engels Vita: Schmidt (2005: 111-115); zu Le Play und seiner Sozialstatistik: Porter (2011); zu Adolphe Quetelet vgl. Prévost \& Beaud (2012: Kapitel 3).

10 Viele Themen der Teilnehmer, so Nico Randeraad, seien patriotisch inspiriert und schwer zu für eine genuin internationale Agenda zu gewinnen gewesen (vgl. Randeraad 2011: 56). Der Austausch über Technikalitäten der Datenverarbeitung funktionierte demgegenüber meines Erachtens als transnationaler Diskurs.

11 Hacking (1987) und Hacking (1990: 189 f.) Demgegenüber verweist Libby Schweber auf unterschiedliche Traditionen in der beschreibenden Statistik und der politischen Arithmetik auch in England und Frankreich (vgl. Schweber 2006).

12 Dies betrifft insbesondere Schneiders Schlussfolgerung, die zentralen technischen Neuerungen Engels, welche die statistischen Kombinationsmöglichkeiten regelrecht revolutionierten, seien „zum Gutteil nur ein Versprechen“ geblieben und erst mit der Einführung der elektronischen Zählmaschine umfassend umgesetzt worden (vgl. Schneider 2013: 239 und 431 f.).

13 Ebd. Zu einer ähnlich kritischen Einschätzung der konzeptionellen Arbeit des Bureaus kommt Frank Hoffmann in seiner gründlichen quellenkritischen Untersuchung der preußischen Gewerbestatistik zwischen 1816 und 1861, die insbesondere wegen uneinheitlich benutzter Erhebungskriterien und Formulare, wie auch der Vermischung von Erwerbspersonen- und Beschäftigungsstatistik zu Doppelzählungen und anderen erheblichen Unregelmäßigkeiten führte (vgl. Hoffmann 2012).

14 Zur Diskussion der Nützlichkeit von Individual- gegenüber Haushaltsliste (vgl. Zahn 1911).

15 Statistica d'Italia (1867). Popolazione, Parte I: Censimento Generale (31 Dicembre 1861) (1867: 3). Zur Geschichte des italienischen Zensus im Allgemeinen (vgl. Patriarca 1996). 
16 Eine detaillierte Beschreibung der Zählblättchenmethode findet sich in Mayr (1914: $122 \mathrm{f}$.).

17 Die Wohnungsnot im explosionsartig wachsenden Berlin und der mit ihr einhergehende "Mietwucher", der vor allem der Verarmung des unteren Mittelstands und der Arbeiterschaft weiter Vorschub leistete, war Gegenstand heftiger Auseinandersetzungen in der Presse der Zeit; auch Engel widmete dem Missstand eine Studie (vgl. Engel 1873a).

$18 \mathrm{Zu}$ Schwabes wie auch Georg Mayrs Arbeiten im Bereich der graphischen Statistik.

19 Der Platz auf der Zählkarte bot nur begrenzt vielen Erhebungskriterien Platz und setzte so den Rahmen für den Umfang der Zählung. Schneider führt aus, dass die Zählkartenmethode und die von ihr ermöglichte leichtere Kombination von Variablen einerseits dazu ermunterte, immer mehr Kriterien abzufragen; in der Praxis jedoch erzwang die Zählkarte ein politisches Ringen um Prioritäten, das 1895 etwa dazu führte, zugunsten der Erhebung der Arbeitslosenquote auf die Angabe des Geburtsorts zu verzichten. Dies hatte dann zur Folge, dass Wanderungsbewegungen in der Bevölkerung statistisch nicht dokumentiert werden konnten (vgl. Schneider 2013: 280).

20 Dieses Verfahren wurde in England bereits seit 1841 eingesetzt (Brückweh 2015: 84). Es war in Preußen nicht unumstritten, führte aber nach Ansicht des Bureaus vor allem in Städten zu überraschend guten Ergebnissen. Zur Diskussion über die Selbstausfüllung von Zählkarten (siehe Schneider 2013: 236 f.).

21 Engel an den Preußischen Innenminister, 18. Juni 1871, Geheimes Staatsarchiv Preußischer Kulturbesitz (GStA PK) HA I, Rep. 77, Tit. 94, Nr. 132, Bd. 1: Kostenanschlag für die Volkszählung von 1871, S. 36.

22 Das wohl bekannteste Beispiel sind die mit astronomischen Berechnungen im Observatorium der Harvard University beschäftigen Frauen (vgl. Mack 1990; Light 1999; Grier 2005: 72-88); für Kompilationsarbeiten des Zensus wurden Frauen in den USA 1890 nach der Einführung des Hollerithsystems eingesetzt, vor allem als Stanzerinnen von Lochkarten, eine ähnliche Entwicklung beschreibt Higgs für das GRO in London bei der Mechanisierung der Zensuskompilation 1911 (vgl. Higgs 1996: 418).

23 Vgl. Blenck an den preußischen Innenminister, 21. April 1898, GStA PK, I HA, Rep. 77, Tit. 536, Nr. 30, Bd. 1, o. p. Zur Figur des Dispatchers oder "Go-Betweens“ (siehe Krajewski 2010: insb. 187-291).

24 Verzeichnis sämmtlicher Hilfsarbeiter nach dem Stand vom 1. April 1890, GStA PK, I HA, Rep. 77, Tit. 536, Nr. 30, Bd. 1, o. p.

25 Blenck an den Preußischen Innenminister, 21. Juli 1898, GStA PK, I HA, Rep. 77, Tit. 536, Nr. 30, Bd. 1, o. p. Die Beschäftigung von Hauspersonal zu Zeiten der Zählung war so üblich, dass Blenck gegenüber dem Ministerium vorbrachte, generell seien die Lebenshaltungskosten der mit Heimarbeit beauftragten Haushalte höher als normal, weil die lange und anstrengende Arbeit Ehefrauen dazu zwang, Bedienstete einzustellen, um die Geschäfte des Haushalts zu übernehmen, aber auch, um aufwändigere Mahlzeiten zuzubereiten, um die Zähler und Zählerinnen bei Kräften zu halten. Blenck an den Innenminister, 8. Mai 1899, GStA PK, HA I, Rep. 77, Tit. 536, Nr. 30, Bd. 2, o.p.

26 Blenck an den Innenminister, 8. Mai 1899, GStA PK PK, I HA, Rep. 77, Tit. 536, Nr. 30, Bd. 2, o. p.

27 Blenck an den Innenminister, 10. Dezember 1908, GStA PK I HA, Rep. 77, Tit. 536, Nr. 30, Bd. 2, o. p.

28 Blenck an den Innenminister, 18. August 1898, betreffs die den Ehefrauen Friedrich und Seidel 1897/8 übertragenen Hausarbeiten, GStA PK, I HA, Rep. 77, Tit. 536, Nr. 30, Bd. 1, o. p.

29 Entgegnung des Preußischen Statistischen Bureaus v. 10.12.1908 auf eine anonyme Beschwerde über die Arbeitsverteilung bei Volkszählungsarbeiten, GStA PK I HA, Rep. 77, Tit. 536, Nr. 30, Bd. 2, o. p.

30 Blenck an den Minister des Innern, 21. April 1898, betreffend die Petition des Militäranwärters Wilhelm Mittelstädt, GStA PK, I HA, Rep. 77, Tit. 536, Nr. 30, Bd. 1, o.p. 
31 So auch der österreichische Statistiker Heinrich Rauchberg, der 1890 in Wien die Einführung des Hollerith-Systems vorantrieb (vgl. Rauchberg 1890: 104).

32 Aus dem gleichen Grund verwarfen die Statistiker in Frankreich das Hollerith-System nach einem ersten Einsatz für die Volkszählung im Jahr 1896 und entwickelten ihren eigenen mechanischen Apparat, den Classicompteur-Imprimateur, der bis in 1930er Jahre im Einsatz war (vgl. Heide 2009: 152).

33 Eine ausführliche Rekonstruktion dieses Konflikts findet sich bei Schneider (2013: 177-185).

\section{Literatur}

Anderson, Chris 2008. The End of Theory: The Data Deluge Makes the Scientific Method Obsolete. Wired Magazine (23. Juni). URL: https://www.wired.com/2008/06/pbtheory/ (25.09.2017).

Anderson, Margo J. 1988. The American Census: a Social History. New Haven: Yale University Press.

Aronova, Elena, Christine von Oertzen und David Sepkoski 2017. Introduction: Historicizing Big Data. Osiris (32): 1-20.

Becker, Peter und William Clark (Hg.) 2001. Little Tools of Knowledge. Historical Essays on Academic and Bureaucratic Practices, Ann Arbor: University of Michigan Press.

Behrisch, Lars (Hg.) 2006. Vermessen, Zählen, Berechnen. Die politische Ordnung des Raums im 18. Jahrhundert. Frankfurt: Campus.

Behrisch, Lars 2016. Die Berechnung der Glückseligkeit. Statistik und Politik in Deutschland und Frankreich im späten Ancien Régime. Ostfildern: Thorbecke.

Blair, Ann 2010. Too Much to Know. Managing Scholarly Information before the Modern Age. New Haven: Yale University Press.

Blenck, Emil 1892. Die Volkszählung vom 1. December 1890 in Preussen und deren endgültige Ergebnisse. Zeitschrift des königlich preussischen statistischen Bureaus (32): 177-264.

Blenck, Emil 1896. Zum Gedächtnis an Ernst Engel. Ein Lebensbild. Zeitschrift des königlich preussischen statistischen Bureaus (36): 231-238.

Blenck, Emil 1897. Die Berufs- und Gewerbezählung vom 14. Juni 1895 und die damit verbundene landwirtschaftliche Betriebszählung. Zeitschrift des königlich preussischen statistischen Bureaus (37): 203-205.

Blenck, Emil 1905. Das Königlich Preußische Statistische Bureau im ersten Jahrhundert seines Bestehens, 1805-1905. Berlin: Königliches Statistisches Bureau.

Bouk, Dan 2017. The History and Political Economy of Personal Data over the Last Two Centuries in Three Acts. Osiris (32): 85-108.

Bowker, Geoffrey 2000. Biodiversity, Datadiversity. Social Studies of Science (30): 643-683.

Brückweh, Kerstin 2015. Menschen zählen. Wissensproduktion durch britische Volkszählungen und Umfragen vom 19. Jahrhundert bis ins digitale Zeitalter. München: de Gruyter Oldenbourg.

Butte, Wilhelm 1808. Die Statistik als Wissenschaft bearbeitet. Landshut: Thormann.

Campbell-Kelly, Martin und William Aspray 2004. Computer. A History of the Information Machine. Boulder: Westview Press.

Charmentier, Isabelle und Staffan Müller-Wille 2014. Worlds of Paper: An Introduction. Early Science and Medicine (19): 379-397.

Daston, Lorraine 2017. The Immortal Archive. Nineteenth-Century Science Imagines the Future. In: Dies. (Hg.). Sciences in the Archives. Pasts, Presents, Futures. Chicago: University of Chicago Press: 159-183.

Daston, Lorraine und Elizabeth Lunbeck (Hg.) 2011. Histories of Scientific Observation. Chicago: University of Chicago Press.

Desrosières, Allain 2005. Die Politik der großen Zahlen. Eine Geschichte der statistischen Denkweise. Heidelberg: Springer. 
Edwards, Paul N. 2010. A Vast Machine: Computer Models, Climate Data, and the Politics of Global Warming. Boston: The MIT Press.

Engel, Ernst 1861. Die Methoden der Volkszählung. Mit besonderer Berücksichtigung der im preussischen Staate angewandten. Zeitschrift des königlich preussischen statistischen Bureaus (7): 149-212.

Engel, Ernst 1867. Aktenmässige Darstellung der Vorbereitungen zu den statistischen Aufnahmen im December 1867, insbesondere der Volkszählung im preussischen Staate und norddeutschen Bundesgebiete. Zeitschrift des königlich preussischen statistischen Bureaus (7): 263-321.

Engel, Ernst 1868. Nachschrift der Redaktion auf Fabricius, Zur Theorie und Praxis der Volkszählungen. Zeitschrift des königlich preussischen statistischen Bureaus (8): 198.

Engel, Ernst 1870. Die Kosten der Volkszählungen mit besonderer Rücksicht auf die im December 1870 im preussischen Staate bevorstehende Zählung. Zeitschrift des königlich preussischen statistischen Bureaus (10): 33-58.

Engel, Ernst 1873a. Die moderne Wohnungsnoth. Signatur, Ursachen und Abhülfe. Leipzig: Duncker und Humblot.

Engel, Ernst 1873b. Die Verwaltung des königlich preussischen statistischen Bureaus im Jahre 1873. Zeitschrift des königlich preussischen statistischen Bureaus (13): 345-364.

Engel, Ernst 1879. Die Aufgaben des Zählwerks im Jahre 1880. Zeitschrift des königlich preussischen statistischen Bureaus (19): 367-376.

Ernst, Wolfgang 2003. Im Namen von Geschichte. Sammeln - Speichern - Er/zählen. Infrastrukturelle Konfigurationen des deutschen Gedächtnisses. München: Wilhelm Fink Verlag.

Friedrich, Markus, Arndt Brendecke \& Susanne Friedrich (Hg.) 2008. Information in der Frühen Neuzeit. Status, Bestände und Strategien. Münster: LIT.

Grier, David Alan 2005. When Computers Were Human. Princeton: Princeton University Press.

Grimmer-Solem, Erik 2003. The Rise of Historical Economics and Social Reform in Germany, 1864-1894. Oxford/New York: Clarendon Press/Oxford University Press.

Haasis, Lucas und Constantin Rieske (Hg.) 2015. Historische Praxeologie. Dimensionen vergangenen Handelns. Paderborn: Schöningh.

Hacking, Ian 1987. Prussian Numbers, 1860-1882. In: Lorenz Krüger, Lorraine Daston und Michael Heidelberger (Hg.). The Probabilistic Revolution. Bd. 1: Ideas in History. Cambridge, Mass.: The MIT Press: 45-68.

Hacking, Ian 1990. The Taming of Chance. Cambridge, Mass.: Cambridge University Press.

Heesen, Anke te 2005. The Notebook. A Paper-Technology. In: Bruno Latour und Peter Weibel (Hg.). Making Things Public. Boston: MIT Press: 582-589.

Heesen, Anke te 2006. Der Zeitungsausschnitt. Ein Papierobjekt der Moderne. Frankfurt/M.: Fischer Taschenbuch.

Heide, Lars 2008. Punched Cards for Professional European Offices: Revisiting the Dynamics of Information Technology Diffusion from the United States to Europe, 1889-1918. History and Technology (24): 307-320.

Heide, Lars 2009. Punched Card Systems and the Early Information Explosion, 1880-1945. Baltimore: Johns Hopkins University.

Hess, Volker und J. Andrew Mendelsohn 2013. Paper Technology und Wissensgeschichte. $\operatorname{NTM}(21): 1-10$.

Higgs, Edward 1996. The Statistical Big Bang of 1911: Ideology, Technological Innovation, and the Production of Medical Statistics. Social History of Medicine (9): 409-426.

Higgs, Edward 2004. Life, Death and Statistics : Civil Registration, Censuses and the Work of the General Register Office, 1836-1952. Hatfield: Local Population Studies.

Hoffmann, Frank 2012. „Ein den thatsächlichen Verhältnissen entsprechendes Bild nicht zu gewinnen": Quellenkritische Untersuchungen zur preußischen Gewerbestatistik zwischen Wiener Kongress und Reichsgründung. Stuttgart: Steiner.

Klein, Ursula 2003. Experiments, Models, Paper Tools: Cultures of Organic Chemistry in the Nineteenth Century. Stanford: Stanford University Press.

Krajewski, Markus 2002. Zettelwirtschaft. Die Geburt der Kartei aus dem Geiste der Bibliothek. Berlin: Kadmos. 
Krajewski, Markus 2010. Der Diener, Mediengeschichte zwischen König und Klient. Frankfurt/M.: Fischer Verlag.

Krajewski, Markus und Peter Krapp 2011. Paper Machines. About Cards E Catalogs, 1548-1929. Cambridge, Mass.: MIT Press.

Krämer, Fabian 2014. Ein Zentaur in London. Lektüre und Beobachtung in der frühneuzeitlichen Naturforschung. Affalterbach/Württ: Didymos-Verlag.

Krug, Leopold 1807. Ideen zu einer staatswirtschaftlichen Statistik. Berlin.

Latour, Bruno 1987. Science in Action: How to Follow Scientists and Engineers through Society. Cambridge, Mass.: Cambridge University Press.

Lemov, Rebecca 2017. Anthropology's Most-Documented Man, c. 1947: A Prefiguration of Big Data from the Big Social Science Era. Osiris (32): 21-42.

Leonelli, Sabina 2008. Bio-Ontologies as Tools for Integration in Biology. Biological Theory (3): 8-11.

Leonelli, Sabina 2016. Epistemische Diversität im Zeitalter von Big Data. Wie Dateninfrastrukturen der biomedizinischen Forschung dienen. In: André Blum, Nina Zschocke, Hans-Jörg Rheinberger und Vincent Barras (Hg.). Diversität: Geschichte und Aktualität eines Konzepts. Würzburg: Königshausen und Neumann: 85-106.

Light, Jennifer 1999. When Computers were Women. Technology and Culture (40): 455-483.

Mack, Pamela 1990. Strategies and Compromises: Women in Astronomy at Harvard College Observatory. Journal of the History of Astronomy (21): 65-75.

Mayr, Georg von 1893. Meine Kritik der preussischen Volkszählungsformulare. Allgemeines Statistisches Archiv (3): 164-182.

Mayr, Georg von 1902. Das Zählblättchen und der britisch-indische Zensus vom 1901. Allgemeines Statistisches Archiv (6): 171-176.

Mayr, Georg von 1914. Statistik und Gesellschaftslehre. Tübingen: Mohr (Paul Siebeck).

Meinel, Christoph 1995. Enzyklopädie des Welt und Verzettelung des Wissens: Aporien der Empirie bei Joachim Jungius. In: Franz Eybl, Wolfgang Harms, Hans-Henrik Krummacher und Werner Welzig (Hg.). Enzyklopädien der Frühen Neuzeit. Beiträge zu ihrer Erforschung. Tübingen: Mohr Siebeck: 160-187.

Müller-Wille, Staffan 2017. Names and Numbers: „Data“ in Classical Natural History, 1758-1859. Osiris (32): 109-128.

Müller-Wille, Staffan und Isabelle Charmantier 2012. Natural History and Information Overload: The Case of Linnaeus. Studies in History and Philosophy of the Biological and Biomedical Sciences (43): 4-15.

Nikolow, Sybilla 2001. A.F.W. Crome's Measurements of the „Strength of the State“: Statistical Representation in Central Europe around 1800. In: Judy L. Klein und Mary S. Morgan (Hg.). The Age of Economic Measurement. Durham: Duke University Press: 23-56.

Nikolow, Sybilla. 1994. Statistiker und Statistik. Zur Genese der statistischen Disziplin zwischen dem 18. und 20. Jahrhundert. Diss. Phil., Universtität Dresden.

Oertzen, Christine von 2017. Machineries of Data Power: Manual Versus Mechanical Census Compilation in Nineteenth-Century Europe. Osiris (32): 129-150.

Oertzen, Christine von 2018. At-Home Census Compilation: Paper, Data, and Technologies of Orderliness. In: Carla, Bittel, Elaine Leong und Christine von Oertzen (Hg.). Working With Paper. Gendered Practices in the History of Knowledge. Pittsburgh: The University of Pittsburgh Press (im Erscheinen).

Patriarca, Silvana 1996. Numbers and Numberhood: Writing Statistics in Nineteenth-Century Italy. Cambridge.

Porter, Theodore 1995. The Rise of Statistical Thinking, 1820-1900. Princeton: Princeton University Press.

Porter, Theodore 2003. Genres and Objects of Social Inquiry, From the Enlightenment to 1900. In: Theodore Porter und Dorothy Ross (Hg.). The Cambridge History of Science, Bd. 7: The Modern Social Sciences. Cambridge: Cambridge University Press: 11-39.

Porter, Theodore 2011. Reforming Vision: The Engineer Le Play Learns to Observe Society Sagely. In: Lorraine Daston und Elizabeth Lunbeck (Hg.). Histories of Scientific Observation. Chicago: Chicago University Press: 281-302.

Prévost, Jean-Guy und Jean-Pierre Beaud 2012. Statistics, Public Debate, and the State, 1800-1945: A Social, Political, and Intellectual History of Numbers. London: Pickering und Otto. 
Randeraad, Nico 2011. The International Statistical Congress (1853-1876): Knowledge Transfers and their Limits. European History Quaterly (41): 50-65.

Rauchberg, Heinrich 1890. Übersicht über den Stand und die neuesten Fortschritte der Technik auf dem Gebiete der Bevölkerungsstatistik. Allgemeines Statistisches Archiv (1): 99-116.

Rheinberger, Hans-Jörg 2011. Infra-Experimentality: From Traces to Data, from Data to Patterning Facts. History of Science (49): 337-348.

Roberts, Lissa (Hg.) 2011. Centres and Cycles of Accumulation in and around the Netherlands in the Early Modern Period, Berlin: Lit Verlag.

Rosenberg, Daniel 2013. Data Before the Fact. In: Lisa Gitelman (Hg.). „Raw Data“ Is an Oxymoron. Cambridge, Mass.: The MIT Press: 15-40.

Schlözer, Ludwig A. 1804. Theorie der Statistik. Nebst Ideen über das Studium der Politik überhaupt. Göttingen: Vandenhoeck und Rupprecht.

Schmidt, Daniel 2005. Statistik und Staatlichkeit. Wiesbaden: VS Verlag für Sozialwissenschaften.

Schneider, Michael C. 2013. Wissensproduktion im Staat. Das königlich preußische statistische Bureau, 1860-1914. Frankfurt/M.: Campus.

Schwabe, Hermann 1869. Die Resultate der Berliner Volkszählung vom 3. December 1867. Berlin: Kortkampf.

Schweber, Libby 2006. Disciplining Statistics. Demography and Vital Statistics in France and England, 1830-1880. Durham: Duke University Press.

Sepkoski, David 2017. The Database Before the Computer? Osiris (32): 175-201.

Sepkoski, David und Marco Tamborini 2018. An Image of Science: Cameralism, Natural History, and the Visual Language of Statistics in the Nineteenth Century. Historical Studies in the Natural Sciences (4) (im Erscheinen).

Soll, Jacob 2009. The Information Master: Jean-Baptiste Colbert's Secret State Intelligence System. Ann Arbor: University of Michigan Press.

Statistica d'Italia 1867. Popolazione, Parte I: Censimento Generale (31 Dicembre 1861). 1867. Florenz: Tipografia di G. Barbèra.

Tanter, Anton 2007. Ordnung der Häuser, Beschreibung der Seelen: Hausnummerierung und Seelenkonskriptionen in der Habsburger Monarchie. Innsbruck: Studienverlag.

Versammlung der Polytechnischen Gesellschaft 1896. Polytechnisches Centralblatt (57): 124.

Vogel, Jakob 2001. Der Undank der Nation: Die Veteranen der Einigungskriege und die Debatte um ihren „Ehrensold“ im Kaiserreich. Militärgeschichtliche Zeitschrift (60): 343-366.

Yates, Joanne 2005. Structuring the Information Age. Life Insurance and Technology in the Twentieth Century. Baltimore: Johns Hopkins University Press.

Zahn, Friedrich (Hg.) 1911. Die Statistik in Deutschland nach ihrem heutigen Stand: Georg von Mayr bei der Feier seines 70. Geburtstags am 12. Februar 1911 als Ehrengabe dargebracht, München, Berlin: J. Schweitzer.

Zedelmaier, Helmut 2015. Werkstätten des Wissens zwischen Renaissance und Aufklärung. Tübingen: Mohr Siebeck.

\section{Christine von Oertzen}

Max-Planck-Institut für Wissenschaftsgeschichte

Boltzmannstraße 22

14195 Berlin

Deutschland

coertzen@mpiwg-berlin.mpg.de 